months for central nervous system (CNS). NI revealed the disease in 14 cases (34\%). CNS and PNS involvements were respectively found in 27 and 19 cases (six patients had both PNS and CNS). In patients with CNS disorders, headache was the most frequent symptom $(n=11)$. Pyramidal syndrome was found in 9 cases and cerebellar syndrome in one patient. Aseptic meningitis was noted in two cases. Brain MRI was performed in 22 cases and showed abnormalities in $82 \%$ of cases. T2 and flair weighted hyperintensities were periventricular $(n=11)$, subcortical $(n=5)$, frontal $(n=3)$ and parietal $(n=2)$. Myelitis was found in one patient.

Patients with PNS showed paresthesia (92\%), motor deficit (22\%) and decreased tendon reflex (18\%). Sensory-motor neuropathy was described in 4 cases, pure sensory neuropathy in 6 cases (including one case of small fiber neuropathy) and motor neuropathy in one patient. Mononeuropathy multiplex was found in 4 cases and polyradiculoneuritis in one patient. Neuromuscular biopsy was performed in five patients and showed vasculitis in two cases. Trigeminal nerve and cochlear nerve were respectively involved in four and one patients. Antinuclear antibodies, anti-SSA and anti-SSB antibodies were respectively positive in $71 \%, 50 \%$ and $28 \%$ of cases. $\mathrm{NI}$ were treated with corticosteroids and immunosuppressive therapy in respectively $56 \%$ and $41 \%$ of cases. Outcome was good in $48 \%$ of the patients. Comparison of clinical and biological features in patients with and without $\mathrm{NI}$ showed no significant differences, only fatigue was significantly associated to $\mathrm{NI}(45.5 \%$ vs $12.9 \%$; $\mathrm{p}=0.005)$.

Conclusions: In our series, NI prevalence was similar to other groups.

CNS involvements were more frequent than PNS manifestations whereas in other ethnic groups they are less frequent.

Disclosure of Interest: None declared

DOI: 10.1136/annrheumdis-2017-eular.2772

\section{FRI0301 CHARACTERIZATION OF FEVER IN HOSPITALIZED PATIENTS WITH SYSTEMIC LUPUS ERYTHEMATOSUS: LESSONS FROM THE JIANGSU COHORT}

X. Feng ${ }^{1}$, W. Pan ${ }^{2}$, L. Liu ${ }^{3}$, M. Wu ${ }^{4}$, L. Sun ${ }^{1}$ on behalf of Jiangsu Lupus Collaborative Group. ${ }^{1}$ Department of Rheumatology, The Affiliated Drum Tower hospital of Nanjing University Medical School, Nanjing; ${ }^{2}$ Department of Rheumatology, Huaian First People's Hospital, Huaian; ${ }^{3}$ Department of Rheumatology, Xuzhou No. 4 People's Hospital, Xuzhou; ${ }^{4}$ Department of Rheumatology, The 3rd Affiliated Hospital of Suzhou University, Changzhou, China

Background: Fever is one of the main symptoms leading to the admission of patients with systemic lupus erythematosus (SLE) and its causes are usually complicated.

Objectives: To explore the prevalence and clinical characteristics of fever in Chinese SLE patients at the time of their first admission and to find out whether fever is related to a poor prognosis.

Methods: A follow-up study aimed to delineate SLE prognosis had been conducted by our Lupus Collaborative Group under the supervisor of Jiangsu Rheumatology Association to collect the data of patients who had ever recorded first admissions during the 1999-2009 decade (1). Among which, those with admission temperature documented were extracted for the assessment of potential factors associated with fever. The independency of various clinical features linked to fever was established by binary logistic regression analysis, and the relation between fever and patient's survival was determined by Cox regression analysis. Results: Totally 1,348 patients were enrolled, in which 1,049 (77.8\%) had normal temperature, $221(16.4 \%)$ had low and moderate fever and $78(5.8 \%)$ had high fever at the time of their first hospitalization. Compared with those having normal temperature, fever patients were more likely to have short disease duration (RR 0.74), concurrent infection (RR 3.29) and gastrointestinal (RR 1.57) as well as hematological involvements (RR 1.56). High C reactive protein level (RR 2.08) and positive anti-Sm antibody (RR1.55) were the two laboratory factors related to fever at admission. However, age, gender, SLEDAI score or erythrocyte sedimentation rate was not independently associated with fever in this cohort. Cox regression analysis showed that there was no correlation between fever and overall deaths (Figure 1) as well as infection induced deaths.

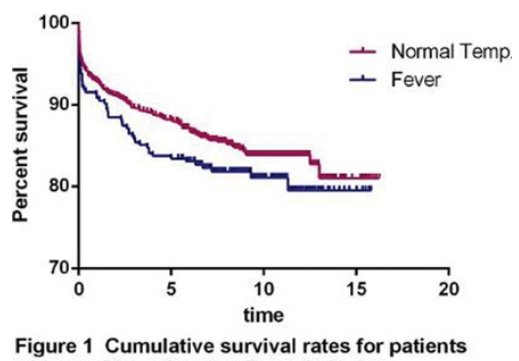

with or without fever at first admission

Conclusions: Both infection and lupus itself (especially gastrointestinal and hematological involvements) contribute to fever in SLE patients. Although the presence of fever increases the complexity of disease treatment, it does not constitute a risk factor for patient's long-term outcome.

\section{References:}

[1] Feng X, Pan W, Liu L, et al. Prognosis for hospitalized patients with systemic lupus erythematosus in China: 5-year update of the Jiangsu cohort. PLoS One. 2016;11(12):e0168619.

Acknowledgements: This study was supported by National Natural Science Foundation of China (81373198) and Jiangsu Provincial Special Program of Medical Science (BE2015602).

Disclosure of Interest: None declared

DOI: 10.1136/annrheumdis-2017-eular.3140

\section{FRI0302 ELEVATED CYSTEINE-RICH PROTEIN 61 IN SYSTEMIC LUPUS ERYTHEMATOSUS-ASSOCIATED PULMONARY ARTERIAL HYPERTENSION}

$\underline{\text { Y. Fan }}{ }^{1}$, J. Qian ${ }^{2}$, Y. Hao ${ }^{1}$, M. Li ${ }^{2}$, L. Gao ${ }^{3}$, X. Zeng ${ }^{2}$, Z. Zhang ${ }^{1} .{ }^{1}$ Departmen of Rheumatology and Clinical Immunology, Peking University First Hospital;

${ }^{2}$ Department of Rheumatology, Peking Union Medical College Hospital, Peking Union Medical College \& Chinese Academy of Medical Sciences, Key Laboratory of Rheumatology and Clinical Immunology, Ministry of Education; ${ }^{3}$ Department of Rheumatology, Beijing Shijitan Hospital, Capital Medical University, Beijing, China

Background: Pulmonary arterial hypertension (PAH) is a complex and devastating complication of connective tissue diseases that leads to severe morbidity and mortality.Unlike in Caucasians, it is systemic lupus erythematosus that recognized as the the major underlying cause of CTD associated PAH in Asian countries, especially in China [1]. Early diagnosis and intervention is vital for better long-term outcome in SLE-PAH patients.Previous study has demonstrated Cysteine-rich protein 61 (Cyr61) was highly expressed in SLE patients [2]. However,the role of Cyr61 in pulmonary arterial hypertension $(\mathrm{PAH})$ remains unknown.

Objectives: To explore the value of Cyr61 for PAH in SLE patients by comparing the plasma Cyr61 levels in SLE patients with/without PAH.

Methods: Plasma samples from two tertiary medical centers were obtained from 54 patients with definite SLE-PAH, 52 age,gender and SLEDAI matched SLE patients without $\mathrm{PAH}$, and 54 age and gender matched healthy controls. Plasma Cyr61 concentration was measured by enzyme-linked immunosorbent assay. Results: Plasma Cyr61 concentration in SLE-PAH patients was significantly higher than the matched SLE patients and healthy controls (median (IQR): 172.5 (143.8, 218.2), 124.9 (104.1, 154.7), 58.17 (28.9, 80.4) respectively, $\mathrm{P}<0.001$ ) (Figure1). The sensitivity and specificity of Cyr61 in predicting the presence of PAH in entire SLE patients were $79.6 \%$ and $67.3 \%$. Receiver operating characteristic curve analysis showed the area under the curve was 0.757 (95\% Cl: 0.662-0.852), with $140.6 \mathrm{pg} / \mathrm{ml}$ as the cut off concentration (Figure2). Further multivariate logistic regression analyses revealed high Cyr61 level $(>140.6)$ is an independent risk factor for SLE patients to develop PAH (OR:7.822 (95\% Cl: 2.224-41.138)) (Table 1). Additionally, weak to moderate positive correlations were observed between Cyr61 concentration and serositis, hematological involvement, red blood cell distribution width, right ventricular systolic pressure and right ventricular diameter measured by echocardiography in entire SLE population.
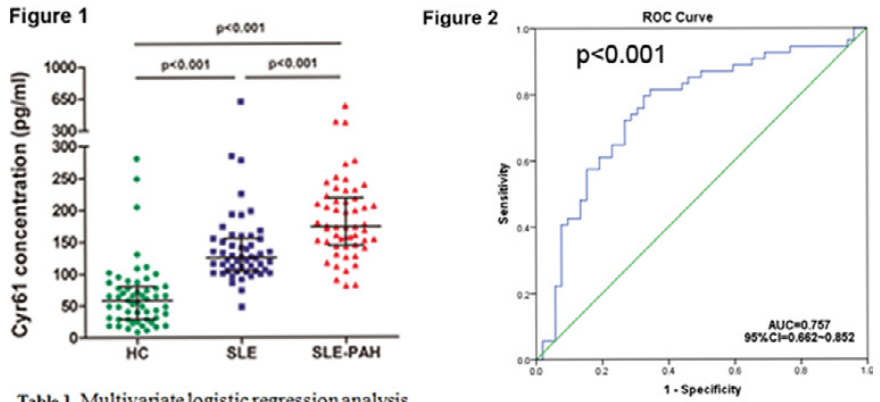

Table 1. Multivariate logistic regression analysis

\begin{tabular}{lcccc}
\hline \multicolumn{1}{c}{ Variables } & OR & \multicolumn{2}{c}{$95 \% \mathrm{CI}$} & P value \\
\hline High Cyr61 level & 7.822 & 2.224 & 41.138 & 0.002 \\
Serositis & 8.244 & 1.854 & 36.663 & 0.006 \\
Anti-RNPpositivity & 3.902 & 1.067 & 14.267 & 0.040 \\
Renal disorders & 0.174 & 0.044 & 0.689 & 0.013 \\
Anti-dsDNA positivity & 0.171 & 0.046 & 0.636 & 0.008 \\
\hline
\end{tabular}

Conclusions: Plasma Cyr61 level was significantly higher in SLE-PAH patients than SLE patients without PAH. Cyr61 may be used as a biomarker for PAH complication in SLE patients.

\section{References:}

[1] Hao, Y.J., et al., Connective tissue disease-associated pulmonary arterial hypertension in Chinese patients. European Respiratory Journal, 2014. 44(4): p. 963-972.

[2] Lin, J., et al., Serum Cyr61 is associated with clinical disease activity and inflammation in patients with systemic lupus erythematosus. Medicine (Baltimore), 2015. 94(19): p. e834 